ARTICLE

\title{
Engineering designer beta cells with a CRISPR-Cas9 conjugation platform
}

\author{
Donghyun Lim¹,2,3, Vedagopuram Sreekanth²,2,3, Kurt J. Cox ${ }^{1,2,3}$, Benjamin K. Law ${ }^{1,2,3}$, Bridget K. Wagner ${ }^{1}$, \\ Jeffrey M. Karp (1) 4,5,6,7 \& Amit Choudhary (1) 1,2,3凶
}

Genetically fusing protein domains to Cas9 has yielded several transformative technologies; however, the genetic modifications are limited to natural polypeptide chains at the Cas9 termini, which excludes a diverse array of molecules useful for gene editing. Here, we report chemical modifications that allow site-specific and multiple-site conjugation of a wide assortment of molecules on both the termini and internal sites of Cas9, creating a platform for endowing Cas 9 with diverse functions. Using this platform, Cas9 can be modified to more precisely incorporate exogenously supplied single-stranded oligonucleotide donor (sSODN) at the DNA break site. We demonstrate that the multiple-site conjugation of ssODN to Cas9 significantly increases the efficiency of precision genome editing, and such a platform is compatible with ssODNs of diverse lengths. By leveraging the conjugation platform, we successfully engineer INS-1E, a $\beta$-cell line, to repurpose the insulin secretion machinery, which enables the glucose-dependent secretion of protective immunomodulatory factor interleukin-10.

\footnotetext{
${ }^{1}$ Chemical Biology and Therapeutics Science Program, Broad Institute of MIT and Harvard, Cambridge, MA 02142, USA. ${ }^{2}$ Department of Medicine, Harvard Medical School, Boston, MA 02115, USA. ${ }^{3}$ Divisions of Renal Medicine and Engineering, Brigham and Women's Hospital, Boston, MA 02115, USA.

${ }^{4}$ Engineering in Medicine, Department of Medicine, Center for Regenerative Therapeutics, Brigham and Women's Hospital, Harvard Medical School, Boston, MA 02115, USA. ${ }^{5}$ Harvard-MIT Division of Health Sciences and Technology, MIT, Cambridge, MA 02139, USA. ${ }^{6}$ Proteomics Platform, Broad Institute of MIT and Harvard, Cambridge, MA 02142, USA. ${ }^{7}$ Harvard Stem Cell Institute, Harvard University, Cambridge, MA 02138, USA.

凶email: achoudhary@bwh.harvard.edu
} 
C lustered regularly interspaced short palindromic repeats (CRISPR)-Cas9 is a DNA endonuclease that can be targeted to a genomic site using a guide RNA (gRNA) bearing sequence complementarity to the target site ${ }^{1}$. The genetic fusion of Cas9 with effector domains (e.g. a transcription activator) has yielded transformative technologies 2,3 ; however, this approach is limited to fusions that are generally linear, polypeptidic, and located on the termini of Cas9. A covalent conjugation platform that allows the creation of fusions that are nonpolypeptidic (e.g. nucleic acids, small molecules, polyethylene glycol [PEG] chains), orthogonally branched from the internal sites of Cas9, and amenable to multiple-site attachment to give a multivalent display of functional cargos would provide a greater diversity of technologies and applications. For example, precise sequence alteration at the Cas9 cleavage site requires the efficient incorporation of exogenously supplied single-stranded oligonucleotide donor DNA (ssODN) ${ }^{4}$ via the homology-directed repair (HDR) pathway,6. However, most cells instead employ the non-homologous end-joining (NHEJ) repair, which results in unpredictable insertions and deletions of bases at the cleavage site, some of which are large enough to have pathogenic consequences ${ }^{7,8}$. Displaying ssODNs on Cas9 can increase their local concentration around the DNA strand break site to allow enhanced incorporation of the desired sequence. In another application, appending PEG chains to Cas9 may reduce the immunogenicity of this bacterial protein ${ }^{9}$.

An ideal conjugation platform to Cas9 should have the following characteristics. First, the platform should be compatible with a diverse set of cargos and allow their multiple-site attachment. Second, the platform should be robust and implementable by nonspecialists given the diverse users of CRISPR technologies. Third, since some of the cargos (e.g. ssODN) are only available in small quantities and are expensive, the conjugation system should work efficiently without requiring large excesses. Ideally, the platform should be modular and inexpensive to allow screening of multiple conditions (e.g. ssODN sequence). Finally, for realworld applications, the platform should allow scaled-up production of the conjugates following good manufacturing practice regulations ${ }^{10}$.

Herein, we present the development of such a platform that relies on thiol-maleimide chemistry and DNA-base pairing, which are both simple, well-established, scalable, and amenable to a wide range of substrates ${ }^{11}$. We systematically scan the domains of Cas9 to choose residues replaceable with engineered cysteines, to which molecules of any size can be efficiently appended without the loss of Cas9 activity. Because many possible conjugates (e.g. ssODNs) are prohibitively expensive for or unamenable to direct thiol-maleimide conjugation, we also seek to develop a more general conjugation platform. Thus, we design a short oligonucleotide handle 'adaptor', which is attached to Cas9 via thiol-maleimide chemistry and uses base pairing to anchor any molecule containing or appended with nucleic acids (Fig. 1a). As an example of the platform's utility, we use it to hybridize long ssODNs that are large, expensive, and not available in sufficient quantities for thiol-maleimide conjugation to Cas9. The resulting Cas9:ssODN conjugates robustly enhance the precise incorporation of the desired sequence from ssODN in multiple cell types and genomic sites. Importantly, the chemical conjugation platform enables the multivalent display of ssODNs, which further enhances the precise incorporation of the desired sequence over that of the unitary display.

Next, we demonstrate the utility of our conjugation platform by efficiently engineering insulin-producing $\beta$ cells to secrete nonendogenous molecules, including an immunomodulatory protein ( $\sim 160$ residues), without incorporating any viral or foreign sequences (e.g. promoter) other than that of the secreted molecule.
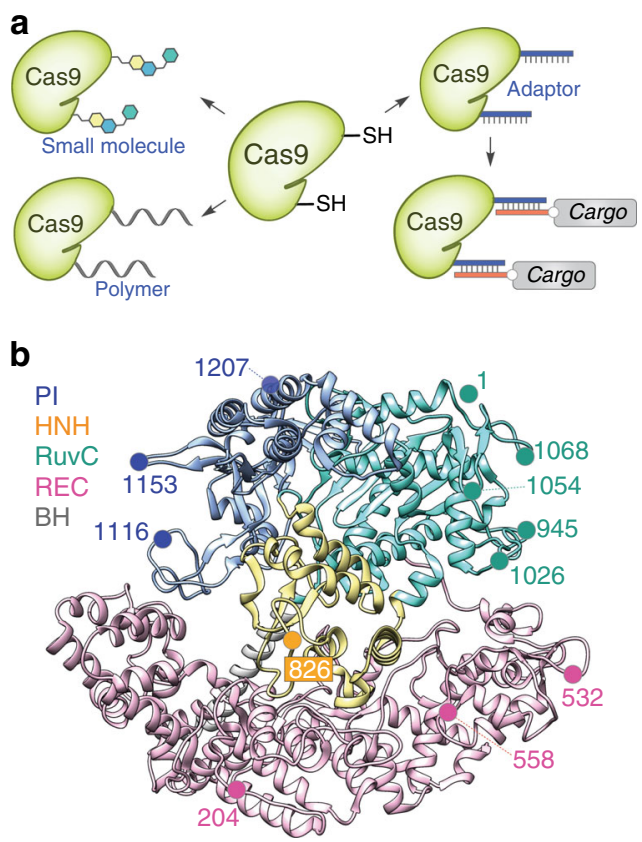

Fig. 1 A conjugation platform for Cas9. a A modular design strategy to functionalize Cas9. b Structure-guided selection of chemical labeling sites. Protospacer adjacent motif-interacting (PI) domain is in blue, $\mathrm{HNH}$ domain is in yellow, RuvC domain is in cyan, recognition (REC) lobe is in magenta, and bridge helix $(\mathrm{BH})$ is in grey. Crystal structure of the Cas9-gRNA-DNA ternary complex is used (PDB: 5F9R) ${ }^{22}$.

Current $\beta$-cell transplantation therapies for type 1 diabetes suffer from immune rejection, resulting in acute cell loss and only shortterm therapeutic effects ${ }^{12,13}$. The macroencapsulation of $\beta$ cells with a semipermeable membrane can protect them from the host's immune system, although foreign body reaction-induced fibrosis can impair the mass transfer and viability of encapsulated cells ${ }^{14-17}$. Anti-inflammatory cytokines, such as interleukin 10 (IL-10), can reduce fibrosis and promote long-term $\beta$-cell survival and superior islet function ${ }^{18-20}$. Therefore, engineered $\beta$-cells that secrete anti-inflammatory cytokines and antifibrotic factors can propel the development of cell-based therapeutics for diabetes. Using our modified Cas9, we genetically repurpose the insulin expression and secretion machinery to secrete a nonendogenous peptide and IL-10 in a glucose-responsive manner, demonstrating an immediate usefulness of our genome editing platform in developing cell-based therapeutics.

\section{Results}

Cas9 domains tolerate the attachment of diverse molecules. To choose the sites for conjugation to Cas9, we analyzed the structures of apo-Cas9, gRNA-bound Cas9, and gRNA- and DNAbound Cas9 for residues that could provide a high labeling yield, tolerate chemical modifications, span all the domains of Cas9, and were surface-exposed in various Cas9 conformations for the efficient modifications (Fig. 1b) $21-23$. Using the aforementioned criteria, we identified two sites $(204,532)$ on the recognition (REC) lobe, one site (826) on the HNH domain, five sites (1, 945, $1026,1054,1068)$ on the RuvC domain, and two sites (1153, 1207 ) on the protospacer adjacent motif-interacting (PI) domain. We selected residues 558 and 1116 as controls, since modifications at 558 will impede the Cas9:gRNA interaction and at 1116 will impede PAM recognition by Cas9 (Fig. $1 \mathrm{~b}$ and Supplementary Fig. 1). We optimized the conjugation conditions for Cas9 variants using biotin-maleimide and PEG $(5 \mathrm{kDa})$-maleimide as model compounds to ensure that modifications of various sizes or 
natures were tolerated (Supplementary Fig. 2). The reactions were fast and high yielding at all sites except for the $1153 \mathrm{C}$ mutantsites proximal to $1153 \mathrm{C}$ (i.e. $1154 \mathrm{C}$ ) also yielded low conjugation efficiencies (Supplementary Fig. 2). The location of these residues was not assigned at the crystal structure of apo-Cas9 but the residues were assumed to be amenable to efficient conjugation since they were expected to be surface-exposed and flexible $e^{21-23}$. Our labeling results, however, indicate that the loop may have higher-order structures that prevent efficient chemical reactions, so we did not use those sites in future experiments. To improve the compatibility of the system with a broader range of conjugates, we next utilized the optimized reaction conditions to label Cas9 at the remaining sites with a 17-nucleotide (nt) DNA adaptor (5'-GCTTCACTCTCATCGTC-3'). The conversion rates were comparable to those of PEG labeling (Supplementary Fig. 2), demonstrating that the efficient conjugation of multiple cargo types can occur at these sites. Thus, the identified sites provide high conjugation yields with diverse molecules, including small molecule and polymers (DNA or PEG).

To identify sites tolerant to the conjugation of the DNA adaptor without the loss of Cas9 activity, we designed an ssODN that would insert a 33-nt DNA fragment (HiBiT sequence $\left.{ }^{24}\right)$ at the target gene (Supplementary Fig. 3). This insertion would result in the expression of a fusion protein with a C-terminal HiBiT tag, which is a small fragment of the NanoLuc luciferase. When HiBiT is complemented by $\mathrm{LgBiT}$, the remainder of NanoLuc, the full-length luciferase is reconstituted to generate a luminescence signal proportional to the degree of knockin, providing an easy readout for HDR (Supplementary Fig. 3a). We chose GAPDH as the first target gene (Supplementary Fig. 3b) owing to its abundant expression in many cell types, which should allow for the reliable detection of the luminescence signal. Using the HiBiT knockin assay, we measured whether appending the DNA adaptor to the cysteine affected Cas9 activity (Fig. 2a). As expected, much of the Cas9 activity was lost by control modifications at residues 558 and 1116, indicating the reliability of the HiBiT knockin assay. We identified five sites whose activity was largely maintained ( $>85 \%$ of wild-type in U2OS), even after labeling with the 17-nt adaptor; these sites stemmed from three regions: the REC lobe (532), the RuvC domain $(1,945,1026)$, and the PI domain (1207). To investigate the off-target profile of the Cas9-adaptor conjugates, we used an eGFP disruption assay with matched gRNA and mismatched gRNAs targeting the eGFP gene of the U2OS.eGFP.PEST cells ${ }^{25,26}$. The Cas9-adaptor conjugate retained the target specificity while also maintaining the on-target activity (Supplementary Fig. $4 a-c$ ). Finally, we demonstrated that Cas9s conjugated to the long PEG chain ( $5 \mathrm{kDa}$, Supplementary Fig. 2c) retained the DNA cleavage activity in the eGFP disruption assay, assuring that Cas9 could be modified with diverse cargos without a loss of function (Supplementary Fig. 4d).

Unitary display of ssODN enhances HDR in several cell types. Next, we designed ssODN with a sequence complementary to the conjugation adaptor and confirmed the binding of the ssODN bearing the complementary sequence to the Cas9-adaptor using a gel-shift assay (Supplementary Fig. 5). To measure the ability of ssODN conjugates to enhance HDR and the site dependence of such enhancements, we performed the HiBiT knockin assay in U2OS cells. Using the luminescence signals from unconjugated ssODN as normalization controls, we observed enhanced knockin efficiency at multiple sites (Fig. 2b and Supplementary Fig. 6a) with the ssODN attached to Cas9. We were able to confirm such enhancements in multiple cell lines, with a greater than four-fold increase in HEK-293FT cells, around a 1.9-fold increase in human-induced pluripotent stem cells, and a more than three-fold increase in primary fibroblasts (Fig. $2 \mathrm{c}-\mathrm{f}$ and Supplementary Fig. $6 \mathrm{~b}-\mathrm{e}$ ). For cells with higher HiBiT signal but lower HDR enhancements, we observed site dependence, with two internal conjugation sites (532, 945) generally performing better than the terminal conjugation a
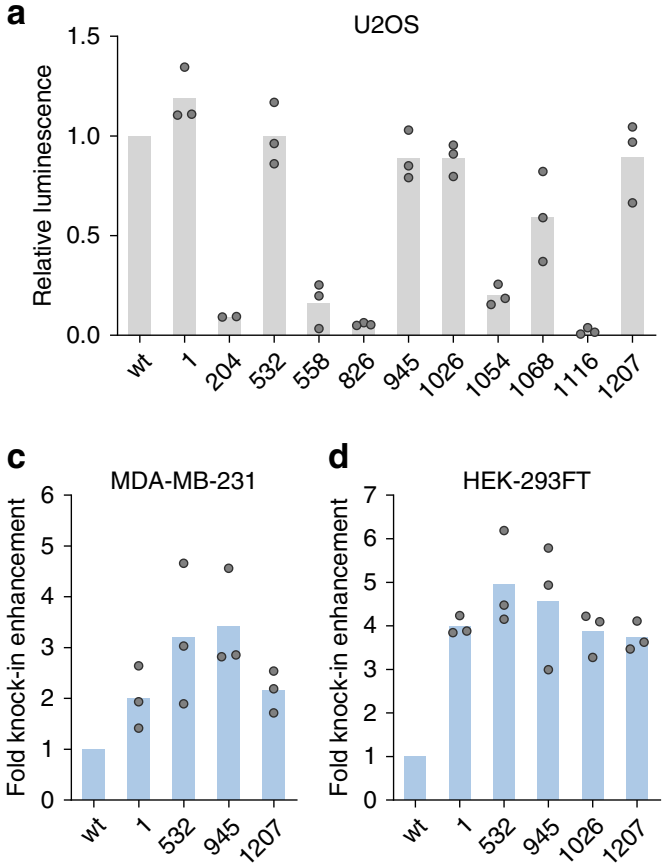
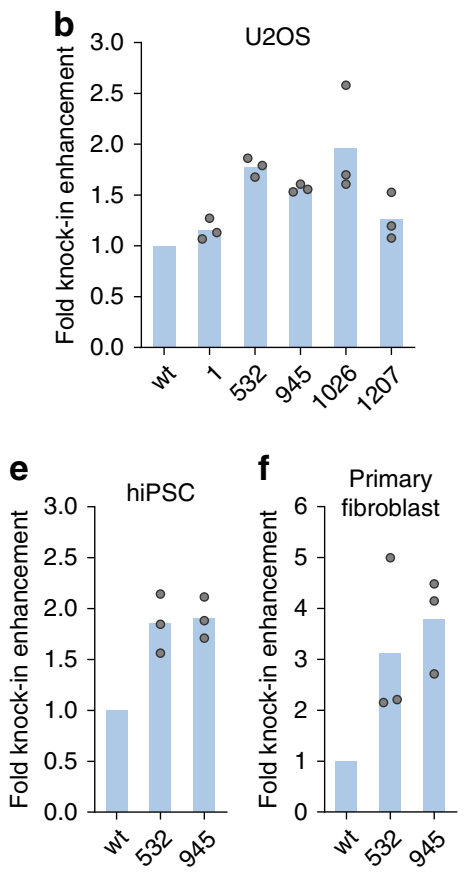

Fig. 2 Unitary display of ssODN on Cas9 domains enhances HDR in multiple cell types. a HiBiT knockin efficiencies by Cas9-adaptor conjugates compared to unlabeled wild-type Cas9 (wt) when a separate Cas9/ssODN system was used ( $n=3$ biologically independent experiments except for 204 where $n=2$ ). b-f ssODN display on Cas9 enhances HiBiT knockin efficiency in various cells: b U2OS, c MDA-MB-231, d HEK-293FT, e human-induced pluripotent stem cells, and $\mathbf{f}$ primary human neonatal dermal fibroblasts. Unlabeled wild-type Cas9 (wt) and Cas9-adaptor conjugates labeled at the indicated residues were used ( $n=3$ biologically independent experiments). Source data are provided as a Source Data file. 
a

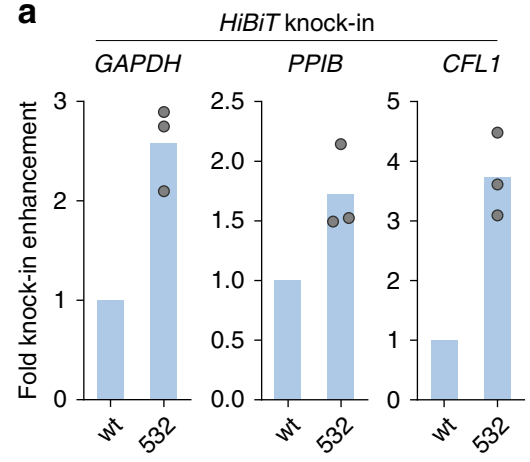

b GFP11 knock-in C

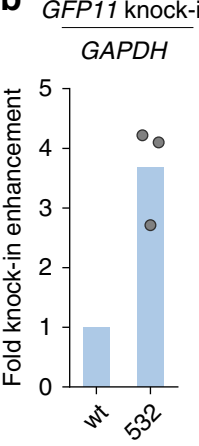

\section{C}

12-base exchange

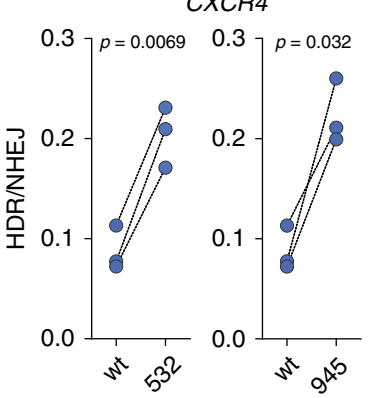

d
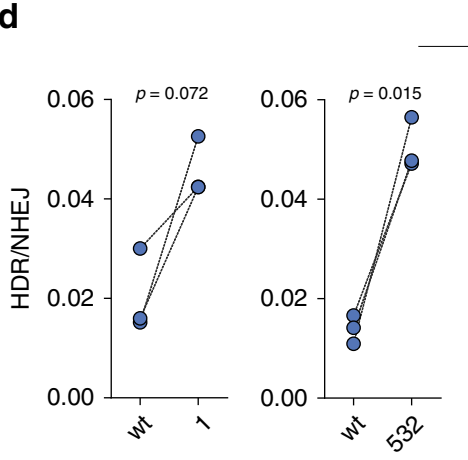

2-base exchange $R B M 20$
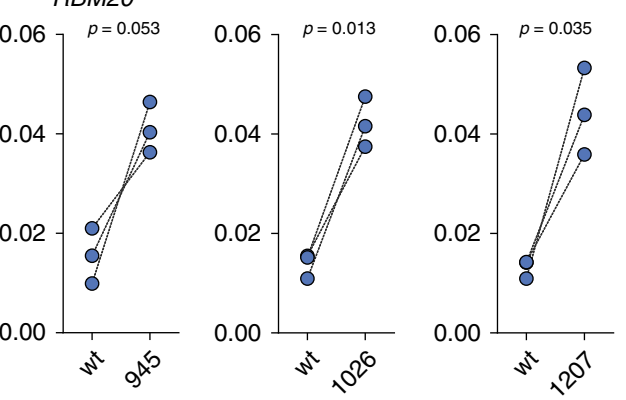

Fig. 3 ssODN display platform allows facile testing of multiple conditions. a HiBiT sequence knockin efficiency was increased at multiple genomic loci in U2OS cells (GAPDH and PPIB) or HEK-293FT cells (CFL1), ( $n=3$ biologically independent experiments). b The GFP11 sequence insertion at the GAPDH locus was promoted in HEK-293T cells ( $n=3$ biologically independent experiments). c HDR-mediated 12-base exchange efficiency at the CXCR4 locus was increased in HEK-293T cells ( $n=3$ biologically independent experiments). d Two-base exchange at the RBM20 locus was promoted in HEK-293FT cells. Unlabeled wild-type Cas9 (wt) and Cas9-adaptor conjugates labeled at the indicated residues were used ( $n=3$ biologically independent experiments). $P$-values were calculated by paired two-tailed $t$-test. Source data are provided as a Source Data file.

site (1). An examination of the crystal structure ${ }^{22}$ indicates that cargos on the two internal residues are expected to align with substrate DNA, while cargos on the terminal residue project outward from the DNA, which may explain the differences in the HDR-enhancing capacities of different ssODN-bearing sites.

ssODN display platform allows rapid and facile screening. To demonstrate the modular nature of our conjugation platform that should allow the rapid testing of multiple conditions and to confirm the generality of HDR enhancement by ssODN display, we tested the ability of the conjugates to enhance HDR under several scenarios (e.g. different genomic sites, ssODN sequences, or readouts). Using the HiBiT knockin assay, we confirmed HDR enhancements at another DNA cleavage site of the GAPDH locus in U2OS (Fig. 3a and Supplementary Fig. 7a) and at multiple genomic loci (PPIB in U2OS, CFL1 in HEK-293FT; Fig. 3a and Supplementary Fig. 7b, c). We then demonstrated HDR enhancement using a fluorescent readout and a longer knockin fragment (GFP11, $57 \mathrm{nt})$. The correct incorporation of this fragment generated detectable fluorescence through the expression of a fusion protein with a C-terminal GFP11 tag, which forms a fully functional GFP when complemented by GFP1-10 (Supplementary Fig. 8) ${ }^{27}$. Here as well, displaying ssODNs on Cas9 increased the knockin efficiency by more than three-fold (Fig. $3 \mathrm{~b}$ and Supplementary Fig. 7d). In addition to luminescence and fluorescence readouts to demonstrate HDR enhancements, we used a restriction endonuclease site knockin assay that quantifies both NHEJ and HDR efficiencies at the CXCR4 locus by gel electrophoresis (Supplementary Fig. 9), and observed the increase in HDR efficiencies by more than two-fold when Cas9: ssODN conjugates were employed (Fig. 3c). We then used a previously reported droplet digital PCR (ddPCR) assay that employs probes to distinguish between wild-type, NHEJ-edited, and HDR-edited sequences at the RBM20 locus (Supplementary Fig. 10a, b) ${ }^{28,29}$. All Cas9:ssODN conjugates increased the ratio of HDR over NHEJ, again indicating the generality of our platform (Fig. 3d). The conjugates also enhanced HDR when another gRNA/ssODN pair was employed (Supplementary Fig. 10c). Finally, we designed an assay to convert eGFP to BFP in $\mathrm{U} 2 \mathrm{OS}$ cells through HDR-based nucleotide exchange and found that Cas9:ssODN conjugates enhanced precision genome editing for this exogenous target gene as well (Supplementary Fig. 11).

Multivalent display of ssODN on Cas9 further enhances HDR. Owing to the small size of our adaptor and the chemical nature of our platform, multivalent displays are feasible (Fig. 4a). To demonstrate multivalent display, we produced Cas9 doublecysteine mutants $(532 \mathrm{C} / 945 \mathrm{C}$ and $532 \mathrm{C} / 1207 \mathrm{C})$ and attached the adaptor to both sites (Supplementary Fig. 12a). Next, we confirmed the binding of the ssODNs to Cas9 (Supplementary Fig. 12b) and observed a boost in HDR efficiency for both the 33nt HiBiT insertion and the two-base exchange (Fig. $4 \mathrm{~b}-\mathrm{d}$ ), indicating that multivalent Cas9 internal modifications further improve the functionality of conjugated Cas9 proteins. Finally, to minimize the size of the labels on Cas9, we investigated the possibility of further decreasing the length of the adaptor. To this effect, we found that hybridization by $13 \mathrm{nt}$ or $15 \mathrm{nt}$ showed a similar HDR-enhancing effect as the standard 17-nt pairing (Supplementary Fig. 13).

Efficient engineering of $\boldsymbol{\beta}$ cells to secrete IL-10. To demonstrate the functional applicability of our chemically modified Cas9, we 


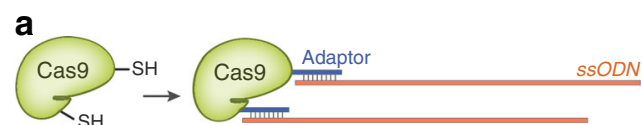

b
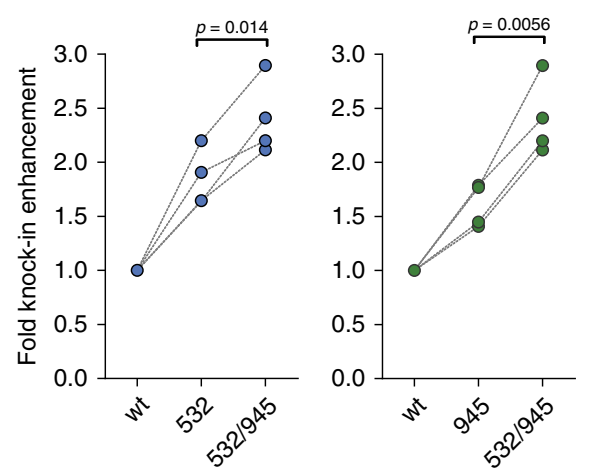

C
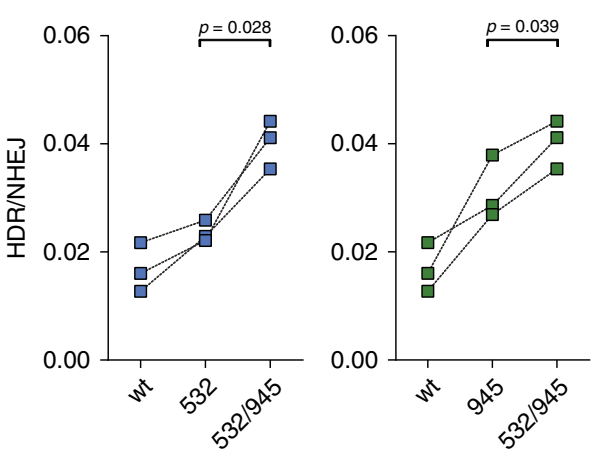

d
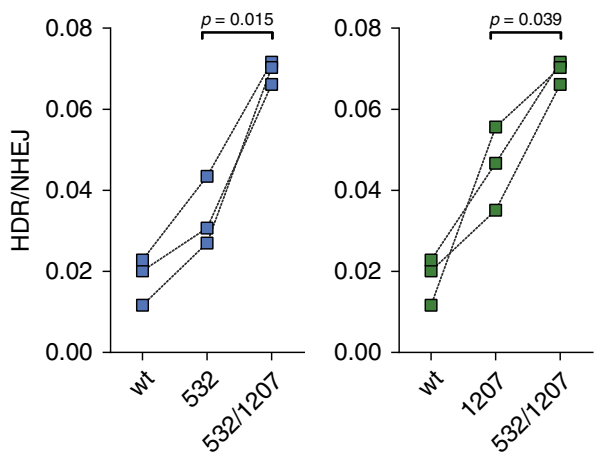

Fig. 4 Multivalent display of ssODN further enhances HDR efficiency.

a Schematic illustrating the production of Cas9 double-ssODN conjugates. b HiBiT sequence knockin at the GAPDH locus was detected in U2OS cells ( $n=4$ biologically independent experiments). c, d Two-base exchange at the RBM20 locus was detected in HEK-293FT cells. Unlabeled wild-type Cas9 (wt) and Cas9-adaptor conjugates labeled at the indicated residues were used ( $n=3$ biologically independent experiments). P-values were calculated by paired two-tailed $t$-test. Source data are provided as a Source Data file.

used it to efficiently engineer $\beta$ cells to endow the cells with immunomodulatory function. Since C-peptide is cleaved during proinsulin processing and is cosecreted with insulin, we hypothesized that knocking in the desired gene into the C-peptide portion of the proinsulin locus would enable the secretion of the inserted gene product. Previously, a lentiviral vector encoding a proinsulin-luciferase fusion construct, containing a luciferase inserted into the C-peptide, expressed functional luciferase in levels directly proportional to insulin when stably integrated into the INS-1E rat $\beta$-cell line and responded sensitively to external stimuli, such as glucose concentration ${ }^{30}$. However, viral-vector engineering poses safety issues such as immunogenicity to viral components or the unintended random insertion of DNA fragments into the host genome $\mathrm{e}^{31,32}$. Direct knockin of the desired gene fragment into the C-peptide locus using Cas9 will allow glucose-dependent cosecretion of the target gene products with insulin. The knockin strategy does not require long regulatory elements (e.g. promoters), which enables footprint-free and efficient HDR because of the smaller knockin size. Any viral or foreign sequences, which are often required to drive efficient gene expression, can be avoided to minimize the immunogenicity issues. In addition, due to the inherent high expression and secretion level of insulin in $\beta$ cells, it would be easy to modulate the secretion level of exogenous gene products (Fig. 5a, b).

We set out to demonstrate our $\beta$-cell engineering strategy with the rat INS-1E $\beta$-cell line, which is widely used to study $\beta$-cell biology. Murine cells have two insulin genes, Ins1 and Ins2, both of which produce and secrete functional insulin; herein, we targeted only the Ins 1 gene. To identify the appropriate insertion site in the Ins1 locus, we used HDR-mediated knockin of the HiBiT sequence at the C-peptide portion (Fig. 5a). The target HiBiT sequence was flanked by additional prohormone convertase 2 (PC2) cleavage sites ${ }^{30}$ to ensure no extra amino acids would be present at each end of the knockin product after processing (Fig. 5a). We chose three gene insertion sites at the start, middle, and terminal regions of the C-peptide locus, and designed several gRNAs to target these sites such that insertion sites and DNA cleavage sites would be close enough to obtain high HDR efficiency (Fig. 5a). In addition, genome-wide offtarget profiles of gRNAs were considered such that potential offtarget sites had mismatches at the seed sequences or at least three mismatches in the gene-encoding regions. When standard genome editing was performed at the target sites using nonconjugated Cas 9 and ssODN, the HiBiT peptide was secreted from INS-1E cells, which could be readily detected through luminescence signals from the cell culture supernatant after complementation by the LgBiT protein. The highest knockin efficiency was achieved by targeting the middle region of the $\mathrm{C}$ peptide (site 2) (Fig. 5c), so this insertion site was used for future experiments. HiBiT peptide secretion was also stimulated by glucose, consistent with the behavior expected from an insertion at the ins1 locus (Supplementary Fig. 14a).

Based on this optimized design, we next knocked in Il10, whose 797-nt ssODN is much larger than that of HiBIT (183 nt). As our approach leverages the insulin secretion pathway, the knockin product will be secreted without the secretory signal peptide. Thus, the signal peptide sequence present in the Il10 gene was omitted when designing the knockin fragment. PC2 cleavage sites were added at each end of Il10 to obtain intact IL-10 as the knockin product, and the corresponding ssODN was synthesized by reverse transcription. When INS-1E cells were transfected with both unconjugated Cas9 and ssODN, IL-10 was secreted to the cell culture medium as determined via enzyme-linked immunosorbent assay (ELISA). No IL-10 was detected after transfection with Cas9 or ssODN alone or in lipopolysaccharide (LPS)-treated cells $^{33}$ (Fig. 5d). We confirmed the correct insertion of the $I l 10$ gene at the Ins1 c-peptide region using Sanger sequencing (Supplementary Fig. 15a-c). Finally, we conjugated ssODN on Cas9 and found that both HiBiT secretion and IL-10 secretion were significantly promoted by Cas9-ssODN conjugation over that of separate Cas 9 and ssODN (Fig. 5e, f and Supplementary Fig. 16).

To further verify that the knockin products are released through the regulated insulin secretion pathway, we investigated the effect of insulin secretagogues ${ }^{30}$ with distinct mode of actions. To minimize the well-to-well signal differences originating from the stochastic distribution of edited cells, we enriched the HiBiT 
a
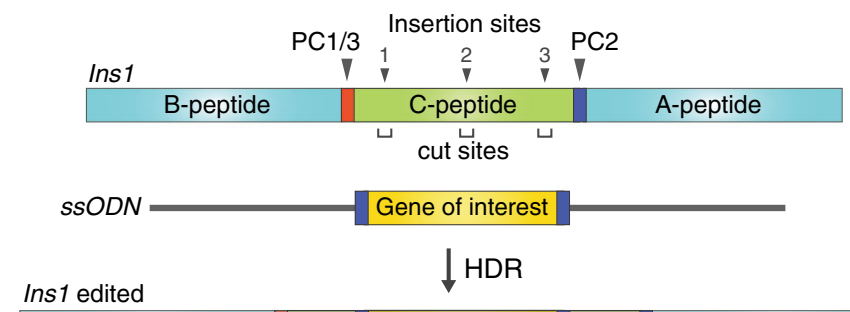

B-peptide Gene of interest

C

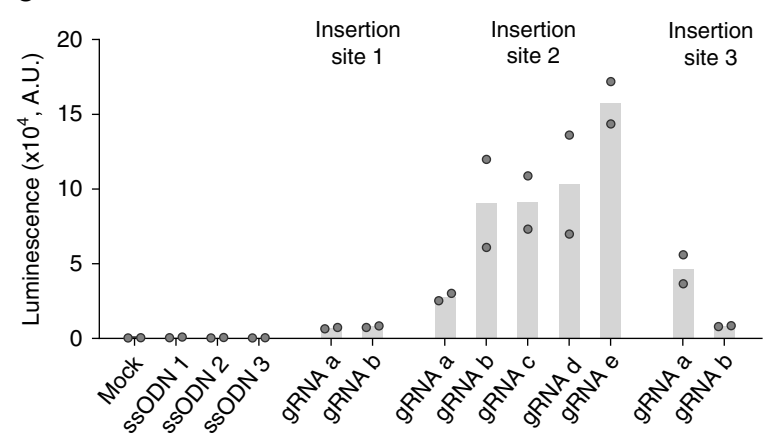

b

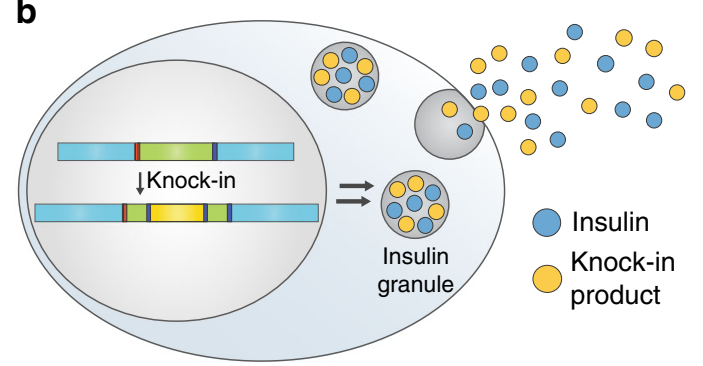

d

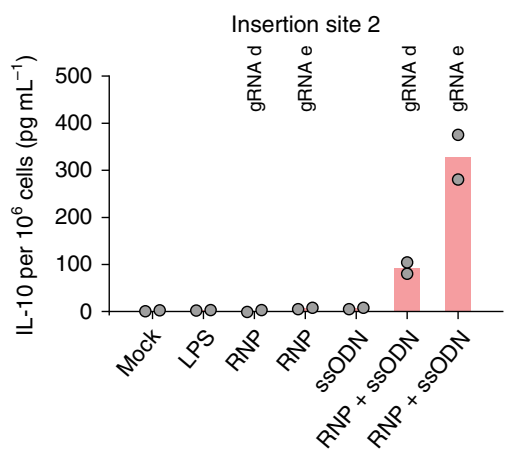

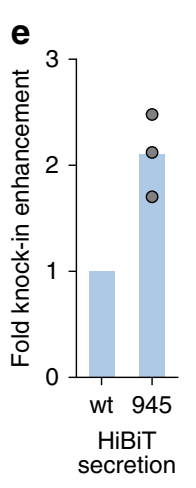

f

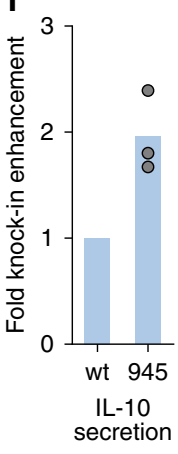

Fig. 5 Efficient engineering of INS-1E cells for secretion of exogenous peptides and proteins. a Schematic of genome editing in the Ins 1 locus of INS-1E cells. PC, prohormone convertase. HDR, homology-directed repair. b Engineered cells can secrete exogenous gene products together with insulin. $\mathbf{c}$ INS-1E cells were engineered to secrete the 11-residue HiBiT peptide. Multiple gene insertion sites and DNA break sites were investigated ( $n=2$ biologically independent experiments). A.U. arbitrary unit. d INS-1E cells were engineered to secrete interleukin 10 (IL-10), ( $n=2$ biologically independent experiments). LPS lipopolysaccharide. RNP ribonucleoprotein. e, $\mathbf{f}$ Display of ssODN on Cas 9 enhanced the secretion of $\mathbf{e}$ HiBiT peptide and $\mathbf{f}$ IL-10 $(n=3$ biologically independent experiments). Source data are provided as a Source Data file.

knockin cells through sib-selection ${ }^{34}$, and these cells were used for the following studies. When the cells were treated with a depolarizing agent, potassium chloride, the HiBiT secretion was increased under both the low- and high-glucose conditions. The use of 3-isobutyl-1-methylxanthine (IBMX), a phosphodiesterase inhibitor that increases the intracellular cAMP level, also increased the HiBiT secretion. Phorbol 12-myristate 13-acetate (PMA), a protein kinase $\mathrm{C}$ activator, induced a large increase in the HiBiT secretion (Fig. 6a and Supplementary Fig. 14c). On the other hand, diazoxide, a compound that opens the potassium channel and hyperpolarizes the $\beta$ cell, decreased the HiBiT secretion at the glucose-stimulated condition (Fig. 6b and Supplementary Fig. 14d). Taken together, the HiBiT signal originating from the endogenous insulin gene should be a reliable surrogate for insulin quantification. Due to the simplicity of HiBiT measurements compared to the insulin ELISA, our HiBiT knockin INS-1E cell line could be used as a reporter to study $\beta$ cell biology when high-throughput methodologies are required.

We then investigated the glucose response of IL-10 secretion after enriching the $I l 10$ knockin cells through sib-selection ${ }^{34}$. As expected from the precise insertion at the Ins1 locus, IL-10 secretion was dependent on the glucose level (Supplementary Fig. 14b). Moreover, the secretion was stimulated by IBMX (Fig. $6 c$ and Supplementary Fig. 14e) and was inhibited by diazoxide (Fig. 6d and Supplementary Fig. 14f). When the knockin cells were incubated with varying concentrations of glucose, insulin and IL-10 secretion was closely correlated (Fig. 6e). Genotyping and a glucose-stimulated IL-10 secretion test indicated that only $2 \%$ of the Ins 1 gene contained Il10 knockin, though these cells still secreted on average $5.4 \mathrm{ng} \mathrm{mL}^{-1}$ of IL-10 per hour under the glucose-stimulated condition (Supplementary Fig. 14b and 15d), which is substantially higher than median effective doses of IL-10 in many in vitro assays ${ }^{35}$.
Thus, we expect that in future therapeutic applications involving human pancreatic $\beta$-like cells, only a small portion of edited cells would need to be used to ensure normal insulin secretion is mostly intact, and it would be possible to fine-tune the secretion level of exogenous gene product.

\section{Discussion}

We describe a simple, scalable, and modular chemical platform for site-specific Cas9 labeling with a wide range of functional molecules to expand Cas9 functionality. We first identified multiple internal residues on Cas9 that can be modified without compromising Cas9 activity in cells, and our use of thiolmaleimide chemistry opens up a variety of site-specific conjugation locations with simple and easy-to-use chemistries. We showed that internal conjugation sites and multivalent conjugations often had improved knockin efficiencies, indicating the need for conjugation systems that are not limited to single modifications of the Cas9 termini. The identified sites could also be used to attach inhibitors of DNA repair pathways for their local inhibition at the site of the Cas9-induced double-strand break. For example, the coadministration of Cas9 with small-molecule inhibitors of the NHEJ pathway can enhance precision editing ${ }^{36}$, but concerns about mutagenesis stemming from genome-wide NHEJ inhibition has limited the utility of such inhibitors ${ }^{37,38}$. Local NHEJ-pathway inhibition at the strand break site through the multivalent display of NHEJ inhibitors on Cas9 itself may allay such concerns. Such local inhibition has been demonstrated for base editors that display peptidic inhibitors of uracil DNA glycosylase for local enzyme inhibition, which improves baseediting efficiencies ${ }^{39}$. Similarly, the local inhibition of p53 pathway activation can increase the efficiency of precision genome editing in primary cells and stem cells where Cas9-induced double-strand breaks lead to apoptosis via this pathway ${ }^{40,41}$. 

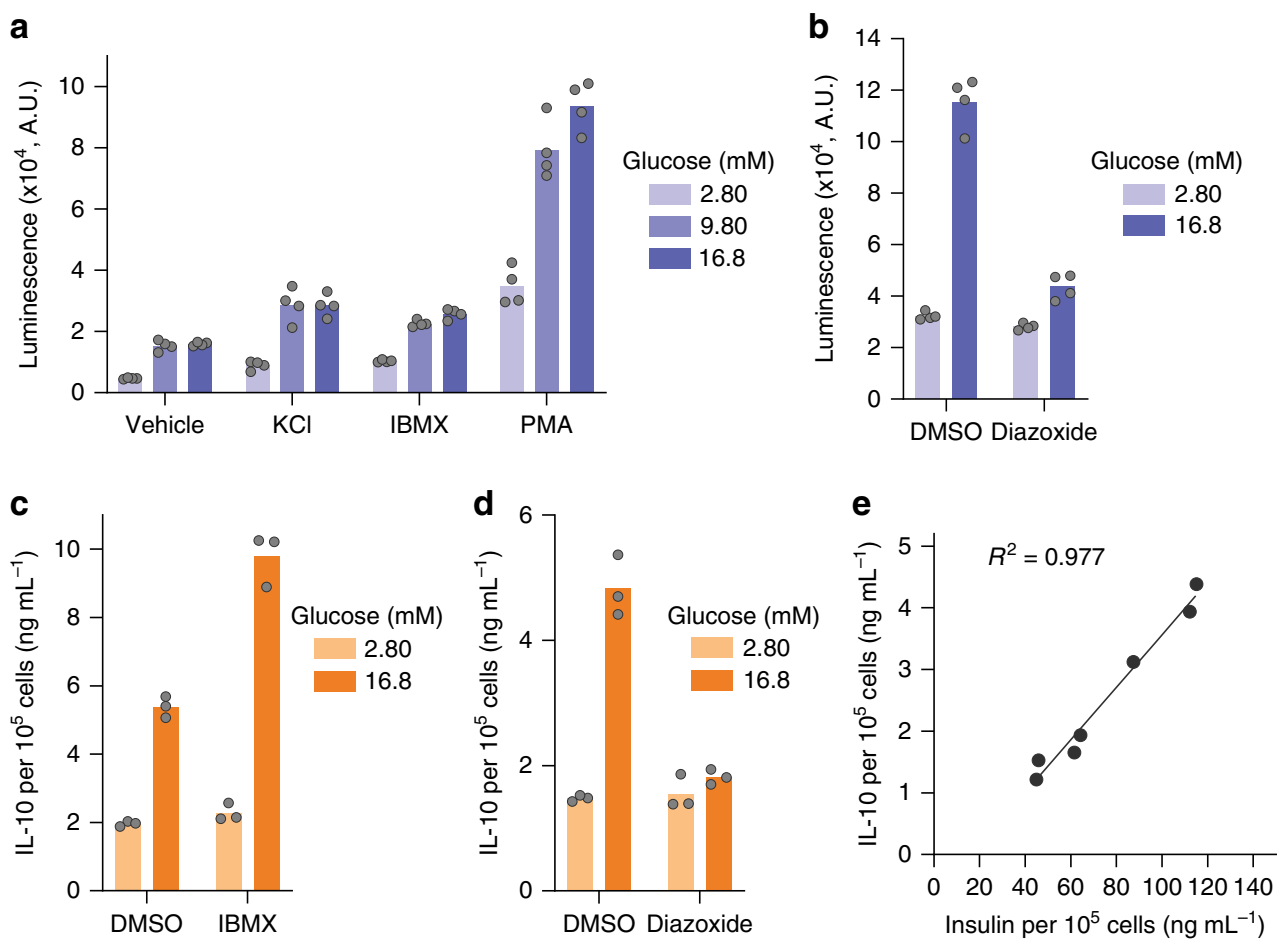

Fig. 6 Knockin products are secreted through the insulin secretion pathway. a, $\mathbf{b}$ Effect of a known insulin secretagogues and $\mathbf{b}$ diazoxide on the HiBiT peptide secretion ( $n=4$ technical replicates). IBMX 3-isobutyl-1-methylxanthine. PMA phorbol 12-myristate 13-acetate. c, d Effect of $\mathbf{c}$ IBMX and d diazoxide on the IL-10 secretion ( $n=3$ technical replicates). e Correlation between insulin secretion and IL-10 secretion under varying glucose concentrations (from $1.40 \mathrm{mM}$ to $16.8 \mathrm{mM}$ ). Data show means of $n=2$ technical replicates. Source data are provided as a Source Data file.

Finally, displaying tissue-specific ligands on Cas9 will enable cellspecific genome editing ${ }^{42}$.

We also developed a short oligonucleotide handle as a universal anchoring point for any oligonucleotide-containing functional molecules, making this platform amenable to most desired conjugates. When ssODN was attached to this anchor, HDR efficiency was enhanced in multiple cells and genomic loci, simultaneously demonstrating the utility of the conjugation technique as well as the usefulness of an increased local concentration of ssODNs for precision genome editing. Our Cas9adaptor design is modular in that the Cas9 bearing the universal adaptor can be used for any ssODN or any type of knockin (short nucleotide exchange, short DNA insertion, and long gene insertion). The use of the optimal ssODN sequence is crucial for successful HDR-based genome editing ${ }^{6}$, and the modular nature of our conjugation platform would allow rapid screening of diverse ssODNs to identify the best one. Even though absolute editing efficiencies are low in some examples (Supplementary Table 7), screening of various ssODN sequences combined with gRNA optimization and modulation of the DNA repair pathways $^{36}$ would enable efficient HDR for practical applications. Finally, the conjugation of ssODNs to Cas9 did not negatively affect cell proliferations, ensuring the usefulness of our conjugation strategy (Supplementary Fig. 17).

While these studies were underway, reports of genetic fusions of Cas9 to avidin, SNAP tag, or porcine circovirus 2 protein (PCV), which can also bind to donor DNAs, appeared in the literature ${ }^{43-46}$, and our studies complement these approaches in multiple ways. First, at 17-nt, our Cas9-adaptor constructs are much smaller than the reported constructs, with the possibility of reducing this to as low as $13 \mathrm{nt}$. Second, while these other genetic fusions are mostly tested at the N- and C-termini of Cas9, we systematically investigated both terminal and internal conjugation sites and found that internal sites yielded higher knockin efficiencies in certain cell lines. The ability of our system to equally address internal or terminal sites makes it significantly more flexible and adaptable to specific applications. Third, our adaptor-based conjugation strategy does not require chemical modification of the ssODNs, as opposed to avidin- or SNAPbased methods, which can be particularly costly and timeconsuming when multiple ssODNs or conditions are being screened during optimization. Fourth, our adaptor sequence can be readily altered to prevent secondary structure formation depending on the ssODN sequence, while the PCV recognition sequence cannot be changed. Finally, owing to the small size of our adaptor and the chemical nature of our platform, multivalent displays are feasible that further enhance HDR and can open up unexplored applications for conjugated Cas9 proteins, expanding the possible range of genome engineering technology.

Strategies for $\beta$-cell genome editing are urgently needed for endowing the cells with required functions, including immunomodulation, to propel the development of cell-based therapeutics for type 1 diabetes ${ }^{14-17}$. Using CRISPR-Cas9 and HDR-based genome editing, we demonstrated that INS-1E cells can be precisely engineered to secret a diverse set of functional molecules, such as small peptides and large gene products. Specifically, we successfully produced cells that cosecrete IL-10, a well-established anti-inflammatory factor that reduces fibrosis and can protect $\beta$ cells from proinflammatory cytokine-induced cell death ${ }^{18-20,47}$. This method should also enable the continuous local production of immunomodulatory factors in $\beta$ cells for preventing $\beta$-cell failure, yet due to its local nature, have minimal systematic effects on the host immune system ${ }^{48}$. Since insulin is secreted in large quantities by $\beta$ cells, only a small fraction of cells may require editing to have a therapeutic effect. Our approach of repurposing the insulin expression and secretion machinery significantly reduces the size of the exogenous sequences that need to be knocked in, allowing efficient engineering of the $\beta$ cells using 
Cas9. Moreover, our precise knockin strategy would be safer than conventional random gene integration methods, which use immunogenic viral vectors that result in unpredictable genomic sequences ${ }^{31,32}$. Because the knockin site was located at the c-peptide region, the knockin product was secreted in a glucosedependent manner, and the secretion level was sensitive to insulin secretagogues. Glucose responsiveness would be advantageous for cells engineered to secrete glucagon-like peptide 1 receptor agonists, which when cosecreted with insulin, would promote insulin secretion and enhance the viability of $\beta$ cells $^{49}$. Additionally, many autoantigens in type 1 diabetes originate from the insulin granule, which may require enhanced immunomodulatory functions under high-glucose conditions ${ }^{50}$.

Overall, this study provides a simple and effective method for forming chemical conjugates of Cas9 to enhance its functionality, and its ease-of-use will make it convenient for scientists of all backgrounds to modify existing Cas9 tools to suit their desired applications. The example application of our method shows that Cas9:ssODN conjugates can successfully enhance precision genome editing in $\beta$ cells, opening up possibilities for chemically enhanced Cas9 in regenerative medicine.

\section{Methods}

Cas9 expression and purification. A plasmid for SpCas9 expression (2x NLS and C-terminal His tag, pET-28a) was a gift from the Gao group (Addgene \#98158) ${ }^{51}$. E. coli Rosette2 (DE3) expressing wild-type Cas9, single-cysteine Cas9 mutants, or double-cysteine Cas9 mutants were grown overnight at $18^{\circ} \mathrm{C}$ with $0.5 \mathrm{mM}$ of IPTG supplemented when the $\mathrm{OD}_{600 \mathrm{~nm}}$ reached $0.8-1.2$. The protein was purified by successive Ni-NTA affinity chromatography, cation exchange chromatography, and size-exclusion chromatography. Purified proteins were snap-frozen in liquid nitrogen and stored at $-80^{\circ} \mathrm{C}$ in Cas 9 storage buffer $(20 \mathrm{mM}$ Tris. $\mathrm{HCl}, 0.1 \mathrm{M} \mathrm{KCl}$, $1 \mathrm{mM}$ TCEP, $20 \%$ glycerol, $\mathrm{pH} 7.5$ ).

Site-directed mutagenesis. Two cysteine residues in SpCas9 (C80, C574) were replaced by serine to give a cysteine-free mutant. Based on this construct, multiple single-cysteine and double-cysteine mutants were generated by introducing cysteines at the designated residues. Mutagenesis was performed using the partial overlapping primer design method or using a Q5 Site-Directed Mutagenesis Kit (New England Biolabs). UCSF Chimera 1.13 was used to analyze the protein structures and choose the mutation sites.

Cas9 labeling by thiol-maleimide conjugation. Adaptor oligonucleotide (GCT TCA CTC TCA TCG TC) modified with protected maleimide (maleimide-2,5dimethylfuran cycloadduct) at the 5' terminus was synthesized by Gene Link. Prior to thiol-maleimide conjugation, the maleimide group was deprotected via retroDiels-Alder reaction by heating the DNA in toluene for $3 \mathrm{~h}$ at $90^{\circ} \mathrm{C}$. Solvent was removed under the reduced pressure, and the resulting DNA in solid form was dissolved in water to give $2 \mathrm{mM}$ solution. Cas9 cysteine mutants $(4 \mu \mathrm{M})$ were mixed with $300 \mu \mathrm{M}$ of PEG $(5 \mathrm{kDa})$-maleimide or adaptor oligonucleotidemaleimide in reaction buffer $(20 \mathrm{mM}$ Tris. $\mathrm{HCl}, 0.1 \mathrm{M} \mathrm{KCl}, 1 \mathrm{mM}$ TCEP, $\mathrm{pH}$ 7.5). The reaction proceeded for $3 \mathrm{~h}$ at room temperature (RT) with mild rotation. The resulting mixture was diluted with a high-salt buffer $(20 \mathrm{mM}$ Tris. $\mathrm{HCl}, 1 \mathrm{M}$ $\mathrm{KCl}, 1 \mathrm{mM}$ TCEP, $20 \%$ glycerol, pH 7.5) and incubated with Ni-NTA agarose beads at $4{ }^{\circ} \mathrm{C}$. The beads were extensively washed with the high-salt buffer to completely remove non-specifically bound oligonucleotide molecules. Labeled Cas 9 was eluted with an elution buffer $(20 \mathrm{mM}$ Tris. $\mathrm{HCl}, 0.1 \mathrm{M} \mathrm{KCl}, 1 \mathrm{mM}$ TCEP, $250 \mathrm{mM}$ imidazole, $10 \%$ glycerol, pH 7.5). Finally, buffer exchange was conducted using Amicon Ultra- $0.5 \mathrm{~mL}$ centrifugal filters with a $100 \mathrm{kDa}$ cut-off (Millipore) to give Cas9-adaptor conjugates in storage buffer $(20 \mathrm{mM}$ Tris. $\mathrm{HCl}, 0.1 \mathrm{M} \mathrm{KCl}, 1 \mathrm{mM}$ TCEP, $10 \%$ glycerol, pH 7.5).

Cas9 biotin labeling and pull-down by streptavidin beads. Cas9 with enhanced specificity [eSpCas9(1.1) ${ }^{52}$ was used for biotin labeling. Cas9 cysteine mutants $(7 \mu \mathrm{M})$ were mixed with $500 \mu \mathrm{M}$ of EZ-Link ${ }^{\mathrm{mM}}$ Maleimide-PEG ${ }_{2}$-Biotin (Thermo) in a reaction buffer $(20 \mathrm{mM}$ Tris $\mathrm{HCl}, 0.1 \mathrm{M} \mathrm{KCl}, 1 \mathrm{mM}$ TCEP, $\mathrm{pH} 7.5)$. The reaction proceeded for $4 \mathrm{~h}$ at room temperature (RT) with mild rotation. Excess compounds were removed by Bio-Gel P-6 columns (Biorad) according to the manufacturer's protocol. Next, 30 pmol of Cas 9 from the above step was incubated with $30 \mu \mathrm{L}$ of Pierce Streptavidin Magnetic Beads (Thermo) overnight at $4{ }^{\circ} \mathrm{C}$. Flow-through was collected and the beads were washed twice with a washing buffer $(20 \mathrm{mM}$ Tris $\cdot \mathrm{HCl}$, $0.15 \mathrm{M} \mathrm{NaCl}, 0.1 \%$ Tween $20, \mathrm{pH} 7.4 ; 300 \mu \mathrm{L}$ ) and once with the reaction buffer $(300 \mu \mathrm{L})$. The beads were heated to $95^{\circ} \mathrm{C}$ for $5 \mathrm{~min}$ in the presence of SDS-PAGE buffer, and the resulting bead-bound fraction (eluate) and flow-through were subjected to SDS-PAGE followed by Coomassie staining.
Electrophoretic mobility shift assay. For this assay, $300 \mathrm{nM}$ of Cas 9 was mixed with $300 \mathrm{nM}$ of ssODNs in a binding buffer $(20 \mathrm{mM}$ Tris. $\mathrm{HCl}, 0.1 \mathrm{M} \mathrm{KCl}, 1 \mathrm{mM}$ TCEP, $10 \%$ glycerol, $\mathrm{pH} 7.5$ ). For the Cas 9 double-adaptor conjugates, $200 \mathrm{nM}$ of protein and $400 \mathrm{nM}$ of ssODN were used. For testing long ssODNs (Supplementary Fig. 16e), $80 \mathrm{nM}$ Cas9 and $60 \mathrm{nM}$ ssODN were used. The mixture was incubated for 30 min at RT and resolved by $1 \%$ agarose gel. DNA was stained using SYBR Gold, and fluorescence images were obtained using an Azure c600 (Azure Biosystems) with the Cy3 channel.

In vitro transcription to synthesize single-guide RNAs. Sequences of targetspecific forward primers and universal reverse primers are listed in Table S2. Polymerase chain reactions (PCRs) were conducted using Q5 High-Fidelity $2 \times$ Master Mix (New England Biolabs) in the presence of $0.5 \mu \mathrm{M}$ of forward and reverse primers in a volume of $25 \mu \mathrm{L}$. The PCR program was as follows: Initial denaturation at $95^{\circ} \mathrm{C}$ for $1 \mathrm{~min} ; 25$ cycles of $95^{\circ} \mathrm{C}$ for $15 \mathrm{~s}, 58^{\circ} \mathrm{C}$ for $30 \mathrm{~s}$, and $72^{\circ} \mathrm{C}$ for $15 \mathrm{~s}$; final extension at $72^{\circ} \mathrm{C}$ for $2 \mathrm{~min}$ and cooling to $25^{\circ} \mathrm{C}$ using a $1 \%$ ramp. The resulting mixture was used for in vitro transcription without purification. The reaction was performed using the HiScribe T7 Quick High Yield RNA Synthesis Kit (New England Biolabs). The mixture contained $10 \mu \mathrm{L}$ of NTP buffer mix, $2 \mu \mathrm{L}$ of the above crude PCR product, $2 \mu \mathrm{L}$ of T7 RNA polymerase mix, and $0.75 \mu \mathrm{L}(30 \mathrm{U})$ of recombinant RNase inhibitor (New England Biolabs) in a final volume of $30 \mu \mathrm{L}$. The reaction was conducted for $12 \mathrm{~h}$ at $37^{\circ} \mathrm{C}$. DNase treatment was performed to remove template DNA according to the manual. RNAs were purified using the MEGAclear Transcription Clean-Up Kit (Invitrogen) according to the manual.

Short single-stranded oligonucleotides. Single-stranded donor DNAs for HiBiT insertion, GFP11 insertion, and nucleotide exchange at the CXCR4 and RBM20 locus were Ultramer DNA oligonucleotides synthesized by Integrated DNA Technology. Their sequences are listed in Supplementary Table 3.

Long single-stranded oligonucleotides for II10 insertion. Single-stranded donor DNAs for Il10 insertion were synthesized by reverse transcription ${ }^{53}$. First, doublestranded gBlocks DNAs were synthesized by Integrated DNA Technology. The DNAs have the T7 promoter sequences followed by the reverse complementary sequences of the final ssODN sequences. DNAs were produced in large quantities by PCR, followed by gel electrophoresis and gel extraction. Next, in vitro transcription was performed using the HiScribe T7 Quick High Yield RNA Synthesis Kit (New England Biolabs). The mixture contained $10 \mu \mathrm{L}$ of NTP buffer mix, $400 \mathrm{ng}$ of the double-stranded DNA template, $2 \mu \mathrm{L}$ of T7 RNA polymerase mix, and $0.4 \mu \mathrm{L}(16 \mathrm{U})$ of recombinant RNase inhibitor (New England Biolabs) in a final volume of $20 \mu \mathrm{L}$. The reaction was conducted for $4 \mathrm{~h}$ at $37^{\circ} \mathrm{C}$. DNase treatment was performed to remove template DNA according to the manual. The resulting RNAs were purified using the MEGAclear Transcription Clean-Up Kit (Invitrogen) according to the manual. Finally, reverse transcription was performed to obtain single-stranded donor DNAs. Approximately 200-250 pmol of RNA was mixed with $400 \mathrm{pmol}$ of reverse primer and $6 \mu \mathrm{L}$ of dNTP mix $(25 \mathrm{mM}$ each, New England Biolabs) in nuclease-free water at a final volume of $35 \mu \mathrm{L}$. The mixture was incubated at $65^{\circ} \mathrm{C}$ for $5 \mathrm{~min}$, then immediately placed on ice for $5 \mathrm{~min}$ to induce RNA-primer annealing. Then, $10 \mu \mathrm{L}$ of $5 \times$ RT buffer $(250 \mathrm{mM}$ Tris $\cdot \mathrm{HCl}, 375 \mathrm{mM}$ $\mathrm{KCl}, 15 \mathrm{mM} \mathrm{MgCl} 2, \mathrm{pH} 8.3), 2.5 \mu \mathrm{L}$ of $0.1 \mathrm{M}$ dithiothreitol solution, $0.5 \mu \mathrm{L}(20 \mathrm{U})$ of RNase inhibitor (New England Biolabs), and $2.5 \mu \mathrm{L}$ of TGIRT-III reverse transcriptase (InGex) were added to the RNA-primer solution. The reaction was proceed at $58^{\circ} \mathrm{C}$ for $3 \mathrm{~h}$. Next, RNA was hydrolyzed by adding $21 \mu \mathrm{L}$ of $0.5 \mathrm{M}$ $\mathrm{NaOH}$ solution and heating at $95^{\circ} \mathrm{C}$ for $10 \mathrm{~min}$. The basic solution was quenched by the addition of $21 \mu \mathrm{L}$ of $0.5 \mathrm{M} \mathrm{HCl}$ solution. The resulting single-stranded DNAs were purified using MinElute PCR Purification Kit (Qiagen) according to the manual. The purity of the ssDNA was confirmed by $6 \%$ TBE-Urea gel electrophoresis followed by SYBR Gold staining. All DNA sequences are listed in Supplementary Tables 4 and 5.

Cell culture. U2OS, MDA-MB-231, HEK-293T, HEK-293FT, and human dermal fibroblasts neonatal (HDFn) cells were maintained in Dulbecco's Modified Eagle Medium (DMEM) supplemented with $10 \%$ fetal bovine serum, $1 \mathrm{mM}$ pyruvate, and penicillin-streptomycin. Human-induced pluripotent stem cells (hiPSC) were maintained on Matrigel in E8 medium. When single-cell passaging of the hiPSC was required, Y-27632 was supplemented at a final concentration of $10 \mu \mathrm{M}$. INS-1E cells were maintained in Roswell Park Memorial Institute (RPMI) medium supplemented with $10 \%$ fetal bovine serum, $1 \mathrm{mM}$ pyruvate, penicillin-streptomycin, and $50 \mu \mathrm{M}$ of 2 -mercaptoethanol.

eGFP disruption assay. Cas9 (10 pmol) and gRNA (10 pmol) were mixed and incubated at RT for $5 \mathrm{~min}$. U2OS.eGFP.PEST cells were transfected with the ribonucleoprotein (RNP) complex using the SE Cell Line 4D-Nucleofector kit (Lonza) following the pulse program of DN-100. After transfection, the cells were suspended in the culture media and transferred to a 96-well plate $(20,000$ cell per well). Forty-eight hours after transfection, the cells were fixed with a $4 \%$ paraformaldehyde solution, and the nuclei were stained with HCS NuclearMask Blue Stain (Invitrogen). The resulting fluorescence signals from eGFP and nuclei were measured using an ImageXpress Micro High-Content Analysis System (Molecular 
Devices) or an Operetta CLS High-Content Analysis System (PerkinElmer). Data acquisition and analysis was performed using MetaXpress (Molecular Devices) or Operetta Harmony 4.8 (PerkinElmer).

HiBiT sequence knockin by nucleofection. U2OS cells stably expressing eGFP. PEST, MDA-MB-231 cells, hiPSC, and HDFn were transfected with Cas9 RNP and ssODN using the cell-specific 4D-Nucleofector kit (Lonza). For U2OS cells, the SE Cell Line kit and the program DN-100 were used. For MDA-MB-231 cells, the SE Cell Line kit and the program CH-125 were used. For hiPSC, the P3 Primary Cell kit and the program CA-137 were used. For HDFn, the P3 Primary Cell kit and the program EN-150 were used. For U2OS and MDA-MB-231 cells, 10 pmol of Cas9, gRNA, and ssODN were used. For hiPSC and HDFn, 20 pmol of Cas9, gRNA, and ssODN were used. For the Cas9:ssODN conjugates, the Cas9-adaptor was premixed with ssODN and incubated at RT for 15-30 min prior to RNP formation to ensure Cas9:ssODN conjugate formation. Then gRNA was added, and the final mixture was incubated for 5-10 min at RT. When Cas9 would not specifically bind ssODNs, the RNP was formed first because it is known that nonspecific Cas9-DNA interactions hamper the RNP formation. After incubating Cas9 and gRNA at RT for 5-10 min, ssODN was added to the mixture. Approximately 200,000 cells (U2OS and MDA-MB-231) or 500,000 cells (hiPSC and HDFn) were transfected with the above mixtures in a well of the nucleofection kit, and 20,000 transfected cells were seeded in each well of a 96-well plate in the case of U2OS and MDA-MB231 cells. For hiPSC and HDFn, all the cells were plated in a well of a 24-well plate. Cells were incubated for $24 \mathrm{~h}$ (U2OS and MDA-MB-231) or $48 \mathrm{~h}$ (hiPSC and $\mathrm{HDFn}$ ) at $37^{\circ} \mathrm{C}$, and cell viability was measured using the PrestoBlue Cell Viability Reagent (Thermo) with SpectraMax M5 and SoftMax Pro 7.0 (Molecular Devices). Next, the HiBiT detection was performed using the Nano-Glo HiBiT Lytic Detection System (Promega) according to the manufacturer's protocol. The luminescence signal was recorded by an EnVision Multilabel Plate Reader with EnVision Manager 1.13 (PerkinElmer). The resulting luminescence signals were normalized based on the cell viability. The same instruments and software were used for all the following $\mathrm{HiBiT}$ knockin assays.

HiBiT sequence knockin by lipofection. HEK-293FT cells were seeded in a 96-well plate at a density of 10,000 cells per well. The next day, Lipofectamine CRISPRMAX (Invitrogen) was used to transfect the cells with Cas9 RNP and ssODN, with final concentrations of $25 \mathrm{nM}$ of both reagents in $110 \mu \mathrm{L}$ of medium per well in a 96-well plate. For Cas9:ssODN conjugates, the Cas9-adaptor was premixed with the ssODN in Opti-MEM (Gibco) and incubated at RT for 15-30 min prior to RNP formation. Next, gRNA was added, and the mixture was incubated for $5-10 \mathrm{~min}$ at RT. Then, Plus reagent (Thermo; $0.17 \mu \mathrm{L}$ per well) was added, and the mixture was incubated for an additional 5 min. Finally, Lipofectamine CRISPRMAX ( $0.3 \mu \mathrm{L}$ per well) in Opti-MEM was added, and the mixture was incubated at RT for $5 \mathrm{~min}$. The final transfection mixture was transferred to each well. In cases where Cas9 did not specifically bind ssODNs, the RNP was first formed by incubating Cas9 and gRNA at RT for 5-10 min in Opti-MEM. Then, Plus reagent $(0.17 \mu \mathrm{L}$ per well) was added, and the mixture was incubated for an additional $5 \mathrm{~min}$. Next, the ssODN was added to the mixture. Finally, Lipofectamine CRISPRMAX ( $0.3 \mu \mathrm{L}$ per well) in Opti-MEM was added, and the mixture was incubated at RT for $5 \mathrm{~min}$. The final transfection mixture was transferred to each well. The transfections were performed in three technical replicates for each biological replicate. For knockin experiments using Cas9 double-ssODN conjugates (Fig. 4), RNP formation was performed first to prevent the nonspecific Cas9ssODN interaction that blocks RNP formation and decreases the genome editing efficiency. Cells were incubated for $24 \mathrm{~h}$ at $37^{\circ} \mathrm{C}$, and cell viability was measured using the PrestoBlue Cell Viability Reagent. Next, the HiBiT detection was performed using the Nano-Glo HiBiT Lytic Detection System. The resulting luminescence signals were normalized based on the cell viability.

GFP11 sequence knockin by lipofection. HEK-293T cells were seeded in a 96-well plate at a density of 8000 cells per well. The next day, Lipofectamine CRISPRMAX was used to transfect the cells with Cas9 RNP (30 nM) and ssODN (30 nM) using the same procedures as described above for the HiBiT knockin assay. Approximately $20-22 \mathrm{~h}$ post transfection, the media was exchanged, and the cells were incubated for an additional 2-4 h. Then, a plasmid encoding for a GFP1-10 fragment (Addgene \#70219, a kind gift from Prof. Bo Huang) ${ }^{27}$ was delivered to the cells using Lipofectamine 2000 (Invitrogen) (120 ng plasmid and $0.4 \mu \mathrm{L}$ Lipofectamine per well). A total of $50 \mathrm{~h}$ after RNP and ssODN transfection, the cells were fixed using $4 \%$ paraformaldehyde. Nuclei were stained by HCS NuclearMask Blue Stain (Invitrogen), and fluorescence images were obtained using the ImageXpress Micro (Molecular Devices) with the DAPI and GFP channels. Data acquisition and analysis was performed using MetaXpress (Molecular Devices).

Knockin of the restriction endonuclease site. HEK-293T cells were seeded in a 24 -well plate at a density of 80,000 cells per well. The next day, Lipofectamine CRISPRMAX was used to transfect the cells with Cas9 RNP (50 nM) and ssODN $(50 \mathrm{nM})$ using the same procedures as described above for the HiBiT knockin, with $1.5 \mu \mathrm{L}$ of Plus reagent and $2.5 \mu \mathrm{L}$ of CRISPRMAX in a final volume of $550 \mu \mathrm{L}$ per well. Forty-eight hours post transfection, genomic DNA was extracted using the
DNeasy Blood \& Tissue Kit (Qiagen). The CXCR4 target site was amplified by a primer pair listed in Supplementary Table 6 with the annealing temperature of $67^{\circ} \mathrm{C}$ and the extension time of $35 \mathrm{~s}$ using Q5 Hot Start High-Fidelity $2 \times$ Master Mix (New England Biolabs). The PCR product was purified using the MinElute PCR Purification Kit (Qiagen). The HDR efficiency was measured by HindIII digestion of $100 \mathrm{ng}$ of PCR product with 10 units of HindIII-HF (New England Biolabs) in CutSmart Buffer for $1 \mathrm{~h}$ at $37^{\circ} \mathrm{C}$. The reaction products were resolved using $1.2 \%$ agarose gel containing SYBR gold (Invitrogen), and gel images were obtained using an Azure c600 (Azure Biosystems). The band intensities were quantified with ImageJ 1.52a, and the percentage of HDR was estimated using the following formula: $100 \times(b+c) /(a+b+c)$, where $a$ is the intensity for the undigested PCR product, and $b$ and $c$ are the intensities for the HindIII cleavage products. NHEJ efficiency was measured by T7EI assay using T7 Endonuclease I (New England Biolabs) based on the methods suggested by the supplier. The reaction products were resolved using $1.2 \%$ agarose gel containing SYBR gold, gel images were obtained using an Azure c600, the band intensity was quantified with Image $1.52 \mathrm{a}$, and the percentage of NHEJ was calculated using the following formula: $100 \times\left\{1-[1-(b+c) /(a+b+c)]^{1 / 2}\right\}$, where $a$ is the intensity for the undigested PCR product, and $b$ and $c$ are the intensities for the T7E1 cleavage products.

Droplet digital PCR-based assay to quantify NHEJ and HDR. HEK-293FT cells were seeded in a 96-well plate at a density of 10,000 cells per well. The next day, the cells were transfected with Cas9 RNP $(35 \mathrm{nM})$ and ssODN ( $35 \mathrm{nM})$ using Lipofectamine RNAiMAX (Invitrogen) in $110 \mu \mathrm{L}$ of media per well in a 96-well plate. For Cas9:ssODN conjugates, the Cas9-adaptor was premixed with ssODN in OptiMEM (Gibco) and incubated at RT for 15-30 min prior to RNP formation. Next, gRNA was added, and the mixture was incubated for $5-10 \mathrm{~min}$ at RT. Finally, Lipofectamine RNAiMAX ( $0.3 \mu \mathrm{L}$ per well) in Opti-MEM was added, and the mixture was incubated at RT for $5 \mathrm{~min}$. The final transfection mixture was transferred to each well. In cases where Cas9 does not specifically bind to the ssODNs, the RNP was first formed by incubating Cas9 and gRNA at RT for $5-10 \mathrm{~min}$ in Opti-MEM. Then, the ssODN was added to the mixture. Finally, Lipofectamine RNAiMAX (0.3 $\mu \mathrm{L}$ per well) in Opti-MEM was added, and the mixture was incubated at RT for $5 \mathrm{~min}$. The final transfection mixture was transferred to each well. For experiments using Cas9 double-ssODN conjugates (Fig. 4), RNP formation was performed first because more ssODN was used in comparison to RNP. Two days post transfection, the cells were harvested, and the genomic DNA was extracted using a DNeasy Blood \& Tissue Kit (Qiagen). Genomic sequences were read by droplet digital PCR ${ }^{29}$. The PCR premixture contained $10 \mu \mathrm{L}$ of ddPCR Supermix for Probes (No dUTP) (Bio-Rad \#1863024), $100 \mathrm{ng}$ of genomic DNA, $900 \mathrm{nM}$ of forward and reverse primers each, $250 \mathrm{nM}$ of reference probe, $250 \mathrm{nM}$ of HDR probe, $250 \mathrm{nM}$ of NHEJ probes, $500 \mathrm{nM}$ of dark probe, and $8 \mathrm{U}$ of HindIII-HF (New England Biolabs) in a $20 \mu \mathrm{L}$ solution. Droplets were generated from the premixture and Droplet Generation Oil for Probes (BioRad) using a QX200 Droplet Generator (Bio-Rad) according to the manufacturer's instruction. Next, droplets were subjected to standard PCR using an Eppendorf Mastercycler Pro thermal cycler with the following program: $95^{\circ} \mathrm{C}$ for $10 \mathrm{~min}(\times 1)$ $94^{\circ} \mathrm{C}$ for $30 \mathrm{~s}$ and $59^{\circ} \mathrm{C}$ for $1 \mathrm{~min}(\times 40) ; 98^{\circ} \mathrm{C}$ for $10 \min (\times 1) ; 50 \%$ ramp rate. Droplets were analyzed using a QX200 Droplet Reader (Bio-Rad) at FAM and HEX channels. Blank transfection served as a control to determine the threshold values to define NHEJ and HDR droplets. FAM++ fractions were identified as HDR population, FAM + and HEX - fractions were identified as NHEJ population, and FAM + and HEX+ fractions were identified as wild-type population ${ }^{29}$. Data acquisition and analysis were performed using QuantaSoft 1.6.6 (BioRad).

Conversion of eGFP to BFP. U2OS.eGFP.PEST cells were transfected with RNP complex and ssODNs using the SE Cell Line 4D-Nucleofector kit (Lonza) following the DN-100 program. First, 30 pmol of Cas9 (or Cas9-adaptor) and 33 pmol of gRNA were mixed and incubated at RT for 5-10 min, then 60 pmol of ssODN was added, and the final mixture was incubated at RT for 15-30 min. After transfection, cells were suspended in the culture medium and transferred to a 96-well plate (20,000 cell per well). Seventy-two hours post transfection, the cells were fixed with a $4 \%$ paraformaldehyde solution, and the nuclei were stained with HCS NuclearMask Deep Red Stain (Invitrogen). The resulting fluorescence signals from eGFP, BFP, and nuclei were measured using an Operetta CLS High-Content Analysis System (PerkinElmer). Data acquisition and analysis was performed using Operetta Harmony 4.8 (PerkinElmer).

HiBiT sequence knockin by nucleofection in INS-1E cells. INS-1E cells were transfected with Cas9 RNP and ssODN using the SF Cell Line 4D-Nucleofector kit (Lonza) following the pulse program of DE-130. For Cas9:ssODN conjugates, 20 pmol of Cas9-adaptor were premixed with $20 \mathrm{pmol}$ of ssODN and incubated at RT for 15-30 min prior to RNP formation to ensure Cas9:ssODN conjugate formation. Then 20 pmol of gRNA was added, and the final mixture was incubated for 5-10 min at RT. In cases where Cas9 did not specifically bind ssODNs, the RNP was formed first because nonspecific Cas9-DNA interactions can hamper the RNP formation. After incubating Cas9 and gRNA at RT for 5-10 min, 20 pmol of ssODN were added to the mixture. Approximately 200,000 cells were transfected 
with the above mixtures in a well of the nucleofection kit, and cells were seeded in a well of a 24-well plate. Cells were incubated at $37^{\circ} \mathrm{C}$ for $48 \mathrm{~h}$, and the supernatant was taken to measure the amount of secreted HiBiT peptide using the Nano-Glo HiBiT Extracellular Detection System (Promega). The resulting luminescence signals were normalized based on the cell viability that was measured using the PrestoBlue Cell Viability Reagent (Thermo).

II10 knockin by nucleofection in INS-1E cells. INS-1E cells were transfected with Cas9 RNP and ssODN using the SF Cell Line 4D-Nucleofector kit following the pulse program of DE-130. For Cas9:ssODN conjugates, 20 pmol of Cas9-adaptor were premixed with $12 \mathrm{pmol}$ of ssODN and incubated at RT for 15-30 min prior to RNP formation to ensure Cas9:ssODN conjugate formation. Then 20 pmol of gRNA was added, and the final mixture was incubated for 5-10 min at RT. In cases where Cas9 did not specifically bind ssODNs, the RNP was formed first because nonspecific Cas9-DNA interactions can hamper the RNP formation. After incubating Cas9 and gRNA at RT for 5-10 min, 12 pmol of ssODN were added to the mixture. Approximately 200,000 cells were transfected with the above mixtures in a well of the nucleofection kit, and the cells were seeded in a well of a 24-well plate in $800 \mu \mathrm{L}$ of the growth medium. The cells were incubated at $37^{\circ} \mathrm{C}$ for $72 \mathrm{~h}$, and the medium was taken to measure the amount of IL-10 accumulated for $72 \mathrm{~h}$ using the IL-10 Rat ELISA Kit (Invitrogen, catalog \# BMS629). LPS was used at the concentration of $10 \mu \mathrm{g} \mathrm{mL}^{-1}$. The absorbance signals were recorded using SpectraMax M5 with SoftMax Pro 7.0 (Molecular Devices).

Glucose-stimulated HiBiT secretion. INS-1E cells from the above genome editing experiments for $\mathrm{HiBiT}$ knockin were grown in a large scale. Then, cells were seeded in multiple wells of a 24 -well plate at a density of 150,000 cells per well. The next day, cell were washed with and incubated in Krebs-Ringer bicarbonate (KRB) buffer $\left(138 \mathrm{mM} \mathrm{NaCl}, 5.4 \mathrm{mM} \mathrm{KCl}, 5 \mathrm{mM} \mathrm{NaHCO}, 2.6 \mathrm{mM} \mathrm{MgCl}_{2}, 2.6 \mathrm{mM}\right.$ $\mathrm{CaCl}_{2}, 10 \mathrm{mM}$ HEPES, pH 7.4, 0.5\% BSA) without glucose for $2 \mathrm{~h}$. The cells in each well were subsequently incubated with different concentrations of glucose (from 2.80 to $16.8 \mathrm{mM}$ ) in the KRB buffer for $1 \mathrm{~h}$. The supernatant was taken from each well to measure the amount of secreted HiBiT peptide using the Nano-Glo HiBiT Extracellular Detection System (Promega). The luminescence signal was recorded using Envision with PerkinElmer EnVision Manager 1.13 (PerkinElmer).

Effect of secretagogues and diazoxide on the HiBiT secretion. INS-1E cells knocked in with $H i B i T$ were enriched through sib-selection ${ }^{34}$, which gives consistent luminescence signal between wells of a 96-well plate. For the enrichment, the mixture of cells were plated in a 96-well plate at a density of 1000 cells per well. When the cells become confluent, the luminescence signal from the HiBiT secretion was recorded. Cells from the wells showing the highest signal were grown on a large scale, and the second round of enrichment was performed at a density of 100 cells per well. Finally enriched cells were used for the secretion assay. Those cells were seeded in a 96-well plate at a density of 40,000 cells per well. The next day, the cells were washed with PBS and were incubated in the KRB buffer containing $2.8 \mathrm{mM}$ of glucose for $1 \mathrm{~h}$. The cells in each well were subsequently incubated in the KRB containing different concentrations of glucose $(2.80 \mathrm{mM}$, $9.80 \mathrm{mM}$, or $16.8 \mathrm{mM}$ ) and vehicle (water and DMSO), $40 \mathrm{mM}$ of $\mathrm{KCl}, 50 \mu \mathrm{M}$ of 3-Isobutyl-1-methylxanthine (IBMX), $20 \mu \mathrm{M}$ of phorbol 12-myristate 13-acetate (PMA), or $250 \mu \mathrm{M}$ of diazoxide for $1 \mathrm{~h}$. The supernatant was taken from each well to measure the amount of secreted HiBiT peptide. The luminescence signal was recorded by Envision with PerkinElmer EnVision Manager 1.13 (PerkinElmer).

Glucose-stimulated IL-10 secretion. INS-1E cells knocked in with Il10 were enriched through sib-selection ${ }^{34}$. The mixture of cells were plated in a 96-well plate at a density of 1,000 cells per well. When the cells become confluent, the IL-10 ELISA was performed using the IL-10 Rat ELISA Kit to identify wells with the highest IL-10 secretion. Cells from those wells were grown in a large scale, and the second round of enrichment was performed at a density of 100 cells per well using IL-10 ELISA as the readout. Finally enriched cells were seeded in a 96-well plate at a density of 50,000 cells per well. The next day, the cells were washed with PBS and were incubated in the KRB buffer containing $2.8 \mathrm{mM}$ of glucose for $1 \mathrm{~h}$. The cells in each well were subsequently incubated with different concentrations of glucose $(2.80-16.8 \mathrm{mM})$ in the KRB buffer for $1 \mathrm{~h}$. When investigating the effect of the compounds, the cells were incubated with glucose $(2.80 \mathrm{mM}$ or $16.8 \mathrm{mM})$ and DMSO, $50 \mu \mathrm{M}$ of IBMX, or $250 \mu \mathrm{M}$ of diazoxide for $1 \mathrm{~h}$. The supernatant was taken to measure the amount of secreted IL-10 using the IL-10 Rat ELISA Kit.

\section{Correlation between insulin secretion and IL-10 secretion. INS-1E cells} knocked in with $I l 10$ were seeded in a 96-well plate at a density of 50,000 cells per well. The next day, the cells were washed with PBS and were incubated in the KRB buffer containing $2.8 \mathrm{mM}$ of glucose for $1 \mathrm{~h}$. The cells in each well were subsequently incubated with varying concentrations of glucose $(1.40,2.80,3.36,4.20$, $5.60,9.80,16.8 \mathrm{mM}$ ) in the KRB buffer for $1 \mathrm{~h}$. The supernatant was taken to measure insulin content using the Rat insulin ELISA kit (APLCO, catalog \# 80-INSRT-E01) and IL-10 content using the IL-10 Rat ELISA Kit.
PCR to amplify the II10 knockin sequence. Genomic DNAs from the wild-type and edited INS-1E cells were extracted using a DNeasy Blood \& Tissue Kit (Qiagen). PCR was performed using $50 \mathrm{ng}$ of genomic DNA, $0.5 \mu \mathrm{M}$ of forward primer, $0.5 \mu \mathrm{M}$ of reverse primer, and Q5 Hot Start High-Fidelity 2x Master Mix (New England Biolabs) in a final volume of $25 \mu \mathrm{L}$. The PCR scheme is shown in Supplementary Fig. 15a. Primer sequences are listed in Supplementary Table 6. The HDR efficiency was measured by resolving the PCR product with $1 \%$ agarose gel containing SYBR gold (Invitrogen) and quantifying the band intensity with ImageJ 1.52a. The NHEJ efficiency was measured by T7EI assay using the same procedures as the above restriction endonuclease site knockin assay at CXCR4.

Data analysis. All calculations and plotting were performed using Excel 2016 (Microsoft) and Prism 8 (GraphPad).

Reporting summary. Further information on research design is available in the Nature Research Reporting Summary linked to this article.

\section{Data availability}

The data generated in this study are provided in the manuscript and the supplementary information, and are available from the corresponding author upon reasonable request. Plasmids from Addgene (\#98158 [https://www.addgene.org/98158], and \#70219 [https:// www.addgene.org/70219]) were used in this study. Structural information from PDB (ID: 5F9R [https://www.rcsb.org/structure/5F9R], 4CMP [https://www.rcsb.org/structure/ 4CMP], and 4ZT0 [https://www.rcsb.org/structure/4ZT0]) was used in this study. Source data are provided with this paper.

Received: 8 August 2019; Accepted: 10 July 2020;

Published online: 13 August 2020

\section{References}

1. Chen, J. S. \& Doudna, J. A. The chemistry of Cas9 and its CRISPR colleagues. Nat. Rev. Chem. 1, 0078 (2017).

2. Wang, H., Russa, M. L. \& Qi, L. S. CRISPR/Cas9 in genome editing and beyond. Annu. Rev. Biochem. 85, 227-264 (2016).

3. Gangopadhyay, S. A. et al. Precision control of CRISPR-Cas9 using small molecules and light. Biochemistry 58, 234-244 (2019).

4. Chen, F. Q. et al. High-frequency genome editing using ssDNA oligonucleotides with zinc-finger nucleases. Nat. Methods 8, 753-755 (2011).

5. Wang, H. X. et al. CRISPR/Cas9-based genome editing for disease modeling and therapy: challenges and opportunities for nonviral delivery. Chem. Rev. 117, 9874-9906 (2017).

6. Bollen, Y., Post, J., Koo, B. K. \& Snippert, H. J. G. How to create state-of-theart genetic model systems: strategies for optimal CRISPR-mediated genome editing. Nucleic Acids Res. 46, 6435-6454 (2018).

7. Yang, Y. et al. A dual AAV system enables the Cas9-mediated correction of a metabolic liver disease in newborn mice. Nat. Biotechnol. 34, 334-338 (2016).

8. Kosicki, M., Tomberg, K. \& Bradley, A. Repair of double-strand breaks induced by CRISPR-Cas9 leads to large deletions and complex rearrangements. Nat. Biotechnol. 36, 765-771 (2018).

9. Charlesworth, C. T. et al. Identification of preexisting adaptive immunity to Cas9 proteins in humans. Nat. Med. 25, 249-254 (2019).

10. Marcq, O. Innovations for Next-Generation Antibody-Drug Conjugates pp 113-161 (Springer International Publishing AG, Cham, 2018).

11. Akkapeddi, P. et al. Construction of homogeneous antibody-drug conjugates using site-selective protein chemistry. Chem. Sci. 7, 2954-2963 (2016).

12. Gamble, A., Pepper, A. R., Bruni, A. \& Shapiro, A. M. J. The journey of islet cell transplantation and future development. Islets 10, 80-94 (2018).

13. Sneddon, J. B. et al. Stem cell therapies for treating diabetes: progress and remaining challenges. Cell Stem Cell 22, 810-823 (2018).

14. An, D. et al. Designing a retrievable and scalable cell encapsulation device for potential treatment of type 1 diabetes. Proc. Natl Acad. Sci. USA 115, E263-E272 (2018).

15. Song, S. \& Roy, S. Progress and challenges in macroencapsulation approaches for type 1 diabetes (T1D) treatment: Cells, biomaterials, and devices. Biotechnol. Bioeng. 113, 1381-1402 (2016).

16. Ellis, C., Ramzy, A. \& Kieffer, T. J. Regenerative medicine and cell-based approaches to restore pancreatic function. Nat. Rev. Gastroenterol. Hepatol. 14, 612-628 (2017)

17. Bochenek, M. A. et al. Alginate encapsulation as long-term immune protection of allogeneic pancreatic islet cells transplanted into the omental bursa of macaques. Nat. Biomed. Eng. 2, 810-821 (2018).

18. Russell, M. A. \& Morgan, N. G. The impact of anti-inflammatory cytokines on the pancreatic beta-cell. Islets 6, e950547 (2014). 
19. Souza, K. L. A., Gurgul-Convey, E., Elsner, M. \& Lenzen, S. Interaction between pro-inflammatory and anti-inflammatory cytokines in insulinproducing cells. J. Endocrinol. 197, 139-150 (2008).

20. Carter, J. D. et al. Viral IL-10-mediated immune regulation in pancreatic islet transplantation. Mol. Ther. 12, 360-368 (2005).

21. Jiang, F. G., Zhou, K. H., Ma, L. L., Gressel, S. \& Doudna, J. A. A Cas9-guide RNA complex preorganized for target DNA recognition. Science 348, 1477-1481 (2015).

22. Jiang, F. et al. Structures of a CRISPR-Cas9 R-loop complex primed for DNA cleavage. Science 351, 867-871 (2016).

23. Jinek, M. et al. Structures of Cas 9 endonucleases reveal RNA-mediated conformational activation. Science 343, 1247997 (2014).

24. Schwinn, M. K. et al. CRISPR-mediated tagging of endogenous proteins with a luminescent peptide. ACS Chem. Biol. 13, 467-474 (2018).

25. Fu, Y. et al. High-frequency off-target mutagenesis induced by CRISPR-Cas nucleases in human cells. Nat. Biotechnol. 31, 822-826 (2013).

26. Maji, B. et al. A high-throughput platform to identify small-molecule inhibitors of CRISPR-Cas9. Cell 177, 1067-1079 (2019).

27. Kamiyama, D. et al. Versatile protein tagging in cells with split fluorescent protein. Nat. Commun. 7, 11046 (2016).

28. Kato-Inui, T., Takahashi, G., Hsu, S. \& Miyaoka, Y. Clustered regularly interspaced short palindromic repeats (CRISPR)/CRISPR-associated protein 9 with improved proof-reading enhances homology-directed repair. Nucleic Acids Res. 46, 4677-4688 (2018).

29. Miyaoka, Y. et al. Systematic quantification of HDR and NHEJ reveals effects of locus, nuclease, and cell type on genome-editing. Sci. Rep. 6, 23549 (2016).

30. Burns, S. M. et al. High-throughput luminescent reporter of insulin secretion for discovering regulators of pancreatic Beta-cell function. Cell Metab. 21, 126-137 (2015).

31. Mingozzi, F. \& High, K. A. Overcoming the host immune response to adenoassociated virus gene delivery vectors: the race between clearance, tolerance, neutralization, and escape. Annu. Rev. Virol. 4, 511-534 (2017).

32. David, R. M. \& Doherty, A. T. Viral vectors: the road to reducing genotoxicity. Toxicol. Sci. 155, 315-325 (2017).

33. Chang, E. Y., Guo, B., Doyle, S. E. \& Cheng, G. Cutting edge: involvement of the type I IFN production and signaling pathway in lipopolysaccharideinduced IL-10 production. J. Immunol. 178, 6705-6709 (2007).

34. Miyaoka, Y. et al. Isolation of single-base genome-edited human iPS cells without antibiotic selection. Nat. Methods 11, 291-293 (2014).

35. Wilbers, R. H. P. et al. Re-evaluation of IL-10 signaling reveals novel insights on the contribution of the intracellular domain of the IL-10R2 chain. PLoS ONE 12, e0186317 (2017)

36. Pawelczak, K. S., Gavande, N. S., VanderVere-Carozza, P. S. \& Turchi, J. J. Modulating DNA repair pathways to improve precision genome engineering. ACS Chem. Biol. 13, 389-396 (2018).

37. Boboila, C. et al. Alternative end-joining catalyzes robust IgH locus deletions and translocations in the combined absence of ligase 4 and Ku70. Proc. Natl Acad. Sci. USA 107, 3034-3039 (2010).

38. Sallmyr, A. \& Tomkinson, A. E. Repair of DNA double-strand breaks by mammalian alternative end-joining pathways. J. Biol. Chem. 293, 10536-10546 (2018).

39. Komor, A. C., Badran, A. H. \& Liu, D. R. Editing the genome without doublestranded DNA breaks. ACS Chem. Biol. 13, 383-388 (2018).

40. Haapaniemi, E., Botla, S., Persson, J., Schmierer, B. \& Taipale, J. CRISPR-Cas 9 genome editing induces a p53-mediated DNA damage response. Nat. Med. 24, 927-930 (2018).

41. Ihry, R. J. et al. p53 inhibits CRISPR-Cas9 engineering in human pluripotent stem cells. Nat. Med. 24, 939-946 (2018)

42. Rouet, R. et al. Receptor-mediated delivery of CRISPR-Cas9 endonuclease for cell-type-specific gene editing. J. Am. Chem. Soc. 140, 6596-6603 (2018).

43. Savic, N. et al. Covalent linkage of the DNA repair template to the CRISPRCas9 nuclease enhances homology-directed repair. elife 7, e33761 (2018).

44. Ma, M. et al. Efficient generation of mice carrying homozygous double-floxp alleles using the Cas9-Avidin/Biotin-donor DNA system. Cell Res. 27, 578-581 (2017).

45. Gu, B., Posfai, E. \& Rossant, J. Efficient generation of targeted large insertions by microinjection into two-cell-stage mouse embryos. Nat. Biotechnol. 36, 632-637 (2018).

46. Aird, EricJ., Amber, K. N. L., St., Martin, Reuben, S. Harris \& Wendy, R. Gordon increasing Cas9-mediated homology-directed repair efficiency through covalent tethering of DNA repair template. Commun. Biol. 1, 54 (2018).

47. van Putten, S. M., Wubben, M., Hennink, W. E., van Luyna, M. J. A. \& Harmsen, M. C. The downmodulation of the foreign body reaction by cytomegalovirus encoded interleukin-10. Biomaterials 30, 730-735 (2009).
48. Bettini, M., Castellaw, A. H., Lennon, G. P., Burton, A. R. \& Vignali, D. A. A. Prevention of autoimmune diabetes by ectopic pancreatic beta-cell expression of interleukin-35. Diabetes 61, 1519-1526 (2012).

49. Rowlands, J., Heng, J., Newsholme, P. \& Carlessi, R. Pleiotropic effects of GLP-1 and analogs on cell signaling, metabolism, and function. Front. Endocrinol. 9, 672 (2018).

50. Burrack, A. L., Martinov, T. \& Fife, B. T. T cell-mediated beta cell destruction autoimmunity and alloimmunity in the context of type 1. Diabetes Front. Endocrinol. 8, 343 (2017).

51. Liang, Z. et al. Efficient DNA-free genome editing of bread wheat using CRISPR/Cas9 ribonucleoprotein complexes. Nat. Commun. 8, 14261 (2017).

52. Slaymaker, I. M. et al. Rationally engineered Cas 9 nucleases with improved specificity. Science 351, 84-88 (2016).

53. Li, H. et al. Design and specificity of long ssDNA donors for CRISPR-based knock-in. Preprint at https://doi.org/https://doi.org/10.1101/178905 (2019).

\section{Acknowledgements}

This work was supported by the Burroughs Wellcome Fund (Career Award at the Scientific Interface), DARPA (BrdiN66001-17-2-4055), and NIH (UC4DK116255, R01 DK113597, HL095722).

\section{Author contributions}

D.L., K.J.C., and A.C. designed the conjugation platform. D.L. (with assistance from B.K.L.) developed the conjugation platform. D.L., V.S., and A.C. designed $\beta$-cell engineering experiments with inputs from B.K.W. and J.M.K., and D.L. and V.S. performed those experiments. D.L. and A.C. wrote the manuscript, which was edited by all the authors.

\section{Competing interests}

D.L. and A.C. are named inventors on Patent application no. PCT/US2020/026264 filed by The Broad Institute to compositions and methods as described in this manuscript. J.M.K. has been a paid consultant for companies including Stempeutics, Sanofi, Celltex LifeVaultBio, Gecko Biomedical, Alivio Therapeutics, Skintifique, Molecular Infusions, Landsdowne Labs, Takeda, Quthero, and Mesoblast (see: https://www.karplab.net/team/ jeff-karp). J.M.K. is also an inventor on a patent that was licensed to Mesoblast. J.M.K holds equity in Frequency Therapeutics, a company that has licensed IP generated by J.M.K. that may benefit financially if the IP is further validated. The interests of J.M.K were reviewed and are subject to management plans overseen by his institution in accordance with their conflict of interest policies. All other authors declare no competing interests.

\section{Additional information}

Supplementary information is available for this paper at https://doi.org/10.1038/s41467 020-17725-0.

Correspondence and requests for materials should be addressed to A.C.

Peer review information Nature Communications thanks Christopher Wright and the other, anonymous, reviewer(s) for their contribution to the peer review of this work.

Reprints and permission information is available at http://www.nature.com/reprints

Publisher's note Springer Nature remains neutral with regard to jurisdictional claims in published maps and institutional affiliations.

Open Access This article is licensed under a Creative Commons Attribution 4.0 International License, which permits use, sharing, adaptation, distribution and reproduction in any medium or format, as long as you give appropriate credit to the original author(s) and the source, provide a link to the Creative Commons license, and indicate if changes were made. The images or other third party material in this article are included in the article's Creative Commons license, unless indicated otherwise in a credit line to the material. If material is not included in the article's Creative Commons license and your intended use is not permitted by statutory regulation or exceeds the permitted use, you will need to obtain permission directly from the copyright holder. To view a copy of this license, visit http://creativecommons.org/ licenses/by/4.0/.

(C) The Author(s) 2020 